\title{
An unexpected difficult airway due to an undiagnosed congenital lingual thyroglossal duct cyst in a neonate with gastrointestinal symptoms: A case report
}

\author{
Yoshiki Kohashi ${ }^{1}$, Tomohiro Yamamoto ${ }^{2}$, Miki Igarashi ${ }^{3}$, and Hironobu Nishimaki ${ }^{3}$ \\ ${ }^{1}$ Nagaoka Red Cross Hospital \\ ${ }^{2}$ Niigata University Graduate School of Medical and Dental Sciences \\ ${ }^{3}$ Niigata City General Hospital
}

November 16, 2021

\begin{abstract}
Lingual thyroglossal duct cysts can be a rare cause of feeding difficulties in infants. Here, we describe a case of an undiagnosed lingual thyroglossal duct cyst in an infant with vomiting and feeding difficulty, who underwent surgery for Hirschsprung's disease and had an unexpected difficult airway during anesthesia induction.
\end{abstract}

An unexpected difficult airway due to an undiagnosed congenital lingual thyroglossal duct cyst in a neonate with gastrointestinal symptoms: A case report

Short running title: Abdominal symptoms due to laryngeal cyst

\section{Article category: Case Report}

Yoshiki Kohashi ${ }^{1}$ (find.the.goal1208@gmail.com)

Tomohiro Yamamoto²(yamatomo@med.niigata-u.ac.jp)

Miki Igarashi ${ }^{3}$ (masui.niigata@gmail.com)

Hironobu Nishimaki ${ }^{3}$ (nishimaki@hosp.niigata.niigata.jp)

1. Division of Anesthesiology, Nagaoka Red Cross Hospital, Niigata, Japan.

2. Division of Anesthesiology, Niigata University Graduate School of Medical and Dental Sciences, Niigata, Japan.

3. Division of Anesthesiology, Niigata City General Hospital, Niigata, Japan.

\section{Correspondence:}

Tomohiro Yamamoto

Division of Anesthesiology, Niigata University Graduate School of Medical and Dental Sciences

1-757, Asahimachi-dori, Chuo ward, Niigata, 951-8510, Japan

Tel: +81-25-2272328, Fax: +81-25-2270790

E-mail: yamatomo@med.niigata-u.ac.jp

Number of words: Abstract: 49; Manuscript body: 985 (including references and figure legends) 


\begin{abstract}
Summary
Lingual thyroglossal duct cysts can be a rare cause of feeding difficulties in infants. Here, we describe a case of an undiagnosed lingual thyroglossal duct cyst in an infant with vomiting and feeding difficulty, who underwent surgery for Hirschsprung's disease and had an unexpected difficult airway during anesthesia induction.
\end{abstract}

\title{
Keywords
}

lingual thyroglossal duct cysts

upper airway obstruction

gastrointestinal symptoms

Hirschsprung's disease

unexpected difficult airway

Pentax Airway Scope ${ }^{\circledR}$

\section{Case description}

Congenital laryngeal cysts usually show symptoms associated with upper airway obstruction. ${ }^{1}$ However, they can be a rare cause of feeding difficulties in infants. ${ }^{1,2}$ Here, we describe a 28 -day-old female infant (height, $51 \mathrm{~cm}$; weight, $2.9 \mathrm{~kg}$ ) who showed symptoms of poor weight gain due to feeding difficulties and post-feeding vomiting. It was thought that Hirschsprung's disease was the cause of the gastrointestinal symptoms shortly after birth. ${ }^{4}$ She was scheduled to undergo a transanal Soave procedure ${ }^{3}$ for Hirschsprung's disease; however, an undiagnosed lingual thyroglossal duct cyst led to unexpected difficult ventilation and intubation while securing the airway during anesthesia induction. Written informed consent for publication was obtained from the parents of the infant.

No respiratory symptoms were identified in the preoperative examination. General anesthesia was slowly induced with sevoflurane and nitrous oxide. Through the established venous line, $15 \mathrm{mg}$ thiamylal, $7 \mu \mathrm{g}$ fentanyl, and $2 \mathrm{mg}$ rocuronium were administered after confirming that assisted ventilation under spontaneous breathing was possible. However, mask ventilation became difficult, and the patient developed hypoxemia and bradycardia; her lowest heart rate was 70 per minute and the saturation of peripheral oxygen $\left(\mathrm{SpO}_{2}\right)$ could not be determined accurately. The support of a senior anesthesiologist was requested. Ventilation improved and $\mathrm{SpO}_{2}$ recovered gradually after the senior doctor provided mask ventilation. However, the glottis was not visible using a Macintosh curved-blade laryngoscope (size 0). Successful intubation using an uncuffed tube with an inner diameter (ID) of $3.0 \mathrm{~mm}$ was achieved only on the sixth attempt by the two anesthesiologists after changing the Macintosh curved-blade laryngoscope (size 0) to a Miller straight-blade laryngoscope (size 0) to lift the epiglottis. The surgery was performed as planned, and the patient was transferred to the neonatal intensive care unit (NICU). She was then extubated on postoperative day (POD) 2. However, the feeding difficulty continued, and prominent stridor developed. The first laryngeal endoscopy on POD 8 indicated laryngomalacia. The patient continued to have poor weight gain, and the inspiratory stridor during sleep worsened. Therefore, a second laryngeal endoscopy was performed on POD 21. A large laryngeal tumor that obstructed the epiglottis from the anterior to the posterior side was detected at the tongue base, with the epiglottis being pulled into the glottis during inspiration (Figure A and B, supplementary video). Computed tomography (CT) showed a cystic mass at the tongue base, and cervical ultrasonography also showed a cyst with smooth margins. This was determined to be a congenital laryngeal cyst.

Laryngeal cystectomy was scheduled on POD 27. Based on the published reports, ${ }^{5}$ we planned to manage the airway with the Pentax Airway Scope ${ }^{\circledR}\left(\right.$ Pentax-AWS ${ }^{\circledR}$, Nihon Kohden, Tokyo, Japan). After establishing an intravenous access in advance, a slow induction was started with sevoflurane and nitrous oxide to preserve spontaneous ventilation, and assisted ventilation with a mask was possible. The glottis and esophagus were 
visible by lifting the epiglottis and the cyst upward with the Pentax-AWS ${ }^{\circledR}$ (Figure C). Therefore, we determined that intubation was possible and $2 \mathrm{mg}$ rocuronium and $8 \mu \mathrm{g}$ fentanyl were administered. The intubation was successfully performed using an uncuffed tube with an ID of $3.5 \mathrm{~mm}$ (Figure D). The cyst remained intact during the intubation manipulation and it was completely resected by the otolaryngologist. The patient was transported to the NICU and extubated three days after the laryngeal cystectomy. Her feeding difficulties gradually improved. A thyroglossal duct cyst was diagnosed based on histopathology. The cyst size was $21 \mathrm{~mm} \times 10 \mathrm{~mm} \times 7 \mathrm{~mm}$, which was significantly larger than that seen on the CT scan. The patient was discharged 20 days after the laryngeal cystectomy.

\section{Learning points}

In the present case, the infant had only gastrointestinal symptoms before the first surgery for Hirschsprung's disease. She did not demonstrate typical symptoms of congenital laryngeal cysts such as inspiratory stridor. Therefore, it was not suspected that the feeding difficulties were due to anything other than Hirschsprung's disease. Congenital laryngeal cysts, which can be a rare cause of feeding difficulties in infants, should be considered as a possible differential diagnosis in patients whose stridor gradually worsens and is accompanied by feeding difficulties. Laryngeal endoscopy, cross-sectional imaging with either CT or magnetic resonance imaging, and cervical ultrasonography are important for diagnosing lingual thyroglossal duct cyst. If the tests indicate a suspected laryngeal cyst, an endoscopic examination should be performed again.

\section{Financial disclosures: None}

\section{Conflicts of interest: None}

\section{Details of authors' contributions:}

- Yoshiki Kohashi: This author performed perioperative anesthesia management, wrote the first draft of the manuscript, and prepared the figures and supplementary video.

- Tomohiro Yamamoto: Corresponding author. This author wrote the first draft of the manuscript and prepared the figures and supplementary video.

- Miki Igarashi: This author helped to write the first draft of the manuscript.

- Hironobu Nishimaki: This author assisted in the perioperative anesthesia management and helped to write the first draft of the manuscript.

\section{References:}

1. Choi YW, Chon JY, Moon HS, Kim JY, Lee JY. Anesthetic management of a neonate with congenital laryngeal cyst. Korean J Anesthesiol. 2012;63:282.

2. Suzuki J, Hashimoto S, Watanabe K, Takahashi K. Congenital vallecular cyst in an infant: case report and review of 52 recent cases. J Laryngol Otol. 2011;125:1199-1203.

3. Langer JC, Minkes RK, Mazziotti MV, Skinner MA, Winthrop AL. Transanal one-stage Soave procedure for infants with Hirschsprung's disease. J Pediatr Surg. 1999;34:148-152.

4. de Lorijn F, Boeckxstaens GE, Benninga MA. Symptomatology, pathophysiology, diagnostic work-up, and treatment of Hirschsprung disease in infancy and childhood. Curr Gastroenterol Rep. 2007;9:245-253.

5. Sugita T, Arisaka H. AirWay Scope for Difficult Ventilation in a Patient With Epiglottic Cyst.Anesth Prog. 2018;65:204-205.

\section{Figure Legends}

Figure: Second laryngeal endoscopy on postoperative day 21 revealed a large laryngeal tumor at the tongue base (A and B). The image via Pentax-AWS ${ }^{(r)}$ while securing the airway (C and D). 
A: During exhalation, a large tumor at the tongue base obstructed the epiglottis from the anterior to the posterior side and the glottis was not visible.

B: During inspiration, the epiglottis was pulled into the glottis.

C: The glottis and esophagus were visible by lifting the epiglottis and the cyst upward.

D: Intubation with an uncuffed tube with an internal diameter of $3.5 \mathrm{~mm}$ was successfully performed using Pentax-AWS ${ }^{(\mathrm{r})}$.

\section{Supplementary Video Legends:}

Supplementary Video: Laryngeal endoscopy on postoperative day 21 revealed a large laryngeal tumor at the tongue base

A large tumor obstructing the epiglottis from the anterior to posterior side at the tongue base, and the epiglottis was pulled into the glottis with inspiration.

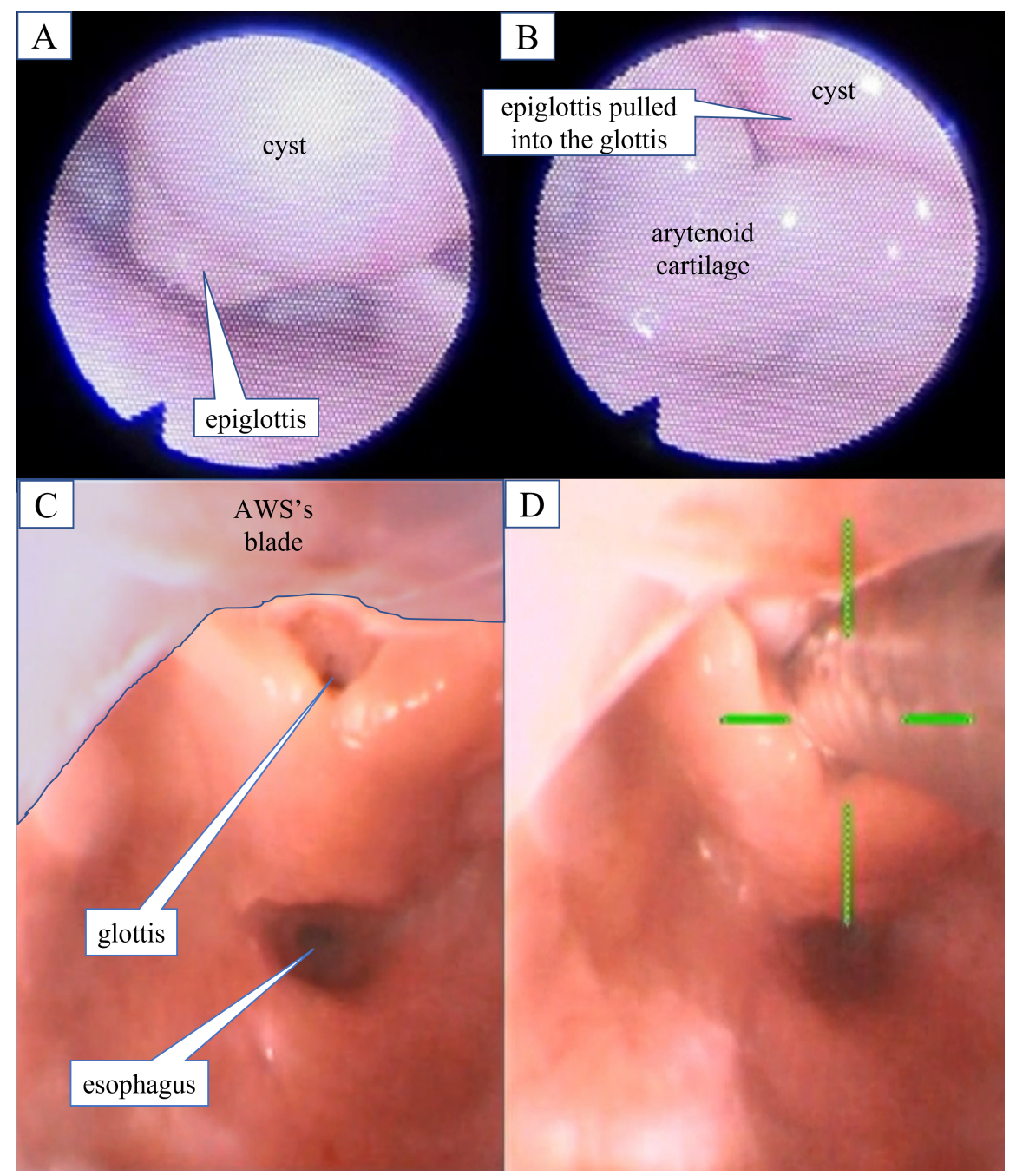

